\title{
Arterial Stiffness in Chronic Kidney Disease: The Usefulness of a Marker of Vascular Damage
}

\author{
Antonio Bellasi, ${ }^{1}$ Emiliana Ferramosca, ${ }^{1}$ and Carlo Ratti ${ }^{2}$ \\ ${ }^{1}$ Division of Nephrology, S.Orsola-Malpighi Hospital, University of Bologna, 40138 Bologna, Italy \\ ${ }^{2}$ Division of Cardiology, Ospedale di Mirandola, 41121 Modena, Italy \\ Correspondence should be addressed to Antonio Bellasi, antoniobellasi@hotmail.com
}

Received 23 March 2011; Accepted 27 March 2011

Academic Editor: Biagio Raffaele Di Iorio

Copyright (c) 2011 Antonio Bellasi et al. This is an open access article distributed under the Creative Commons Attribution License, which permits unrestricted use, distribution, and reproduction in any medium, provided the original work is properly cited.

\begin{abstract}
Increased arterial stiffness is a marker of vasculopathy in chronic kidney disease (CKD) patients, suggesting a significant cardiovascular damage. Detection of arterial stiffness provides physicians with useful prognostic information independent of traditional cardiovascular (CV) risk factors. In addition, this knowledge may help guide appropriate therapeutic choices and monitor the effectiveness of antihypertensive therapies. We review the relationship between arterial stiffness and CKD, as well as the prognostic implications of increased arterial stiffness and the potential therapeutic strategies to ameliorate arterial compliance and outcome in CKD.
\end{abstract}

\section{Introduction}

Traditional CV risk factors are of major importance to identify subjects at risk for cardiovascular disease (CVD). However, in spite of a significant over-representation, traditional CV risk factors do not accurately predict survival in $\mathrm{CKD}$ patients. Hence, several new biomarkers of CV risk and disease have been identified in the hope to refine the CV risk assessment.

It is important to differentiate between circulating and tissue biomarkers. Serum concentration of circulating biomarkers may vary substantially according to the instant risk at which an individual is exposed. Nevertheless, several factors may influence and confound their serum levels, and a recent article questioned the added value of the use of multiple serum biomarkers to refine the risk assessment and outcome prediction [1].

To the contrary, tissue biomarkers such as vascular calcification or arterial stiffness are signs of true tissue damage rather than risk factors. In other words, they reflect the cumulative exposure to damaging factors that have harmed the cardiovascular system. Notably, a growing body of evidence suggests that tissue biomarkers can be useful in combination with traditional CV risk factors to assess the $\mathrm{CV}$ risk of a single individual. Indeed, arterial stiffness or vascular calcification evaluation adds a significant and independent contribution in the prediction of microvascular damage of the heart, brain, retina, and kidney as well as CV outcome.

The usefulness of a biomarker depends on its accuracy in detecting the disease and in guiding treatment. The aim of this paper is to summarize the available evidence that supports the use of arterial stiffness assessment in CKD patients.

\section{Vascular Remodeling and Arterial Stiffness}

Large central arteries play a key role in converting the pulsatile cardiac outflow into a continuous blood flow throughout the arterial tree. In systole, the heart pushes a volume of blood (i.e., stoke volume) into the thoracic aorta (ejection phase). Because fluids are uncompressible, the stroke volume distends the thoracic aorta. By stretching, the arterial wall accumulates the elastic energy that maintains the blood flow during diastole when the ejection phase is over (Windkessel 
effect). Thus, the pulsatile flow is converted into a continuous flow by the cyclic distention and recoiling of central arteries.

The muscular and endothelial cells as well as other components of the arterial wall have to adapt to the different stimuli to which they are exposed to maintain an adequate blood perfusion during diastole. However, aging, increase in the mean arterial pressure, diabetes, or other noxious factors exposure induces vascular structure and function changes that ultimately may lead to arterial wall stiffness increase.

In this regard, a few lines of evidence suggest that exposure to traditional and nontraditional CV risk factors leads to arterial wall remodeling and reduced arterial compliance. All these changes negatively affect the process that converts the pulsatile in continuous blood flow impairing the oxygen supply to peripheral tissues.

Thus, arterial stiffness might be considered as a measure of arterial damage due to the cumulative exposure of aging and different traditional and non-traditional CV risk factors.

\section{Arterial Stiffness and Chronic Kidney Disease: A Vicious Cycle}

Many clinical and epidemiological studies have documented that patients with impaired kidney function are at increased risk of CV events and mortality [2]. Notably, the association of CKD and CV events is significant even with mild decrements of renal function not sufficiently severe to result in elevated serum creatinine [3].

The mechanisms responsible for this strong association are not well established. However, a few lines of evidence suggest that arterial stiffness increases with progressive worsening of renal function. A seminal paper by Wang and coworkers [4] showed a clinically meaningful increase in the aortic pulse wave velocity (PWV), an accurate and reproducible parameter of arterial stiffness, with estimated glomerular filtration rate (eGFR) decline. In this study, while PWV was comparable between age-matched controls and CKD 1-2 patients, there was a significant trend for a stepwise increase in PWV with more advanced stages of CKD (3 through 5). Notably, multivariable analyses confirmed the independent association between eGFR and PWV [4].

This observation was confirmed by other studies. Ix and coworkers [5] demonstrated in a large cross-sectional study of 4,513 individuals from the general population that arterial stiffness assessed via the ankle-brachial index (ABI) was associated with even mild impairments of the renal function (i.e., eGFR below $80 \mathrm{~mL} / \mathrm{min}$ ) estimated by Cystatin-C. In spite of adjustment for age, sex, race, hypertension, diabetes, smoking, body mass index, LDL cholesterol, HDL cholesterol, and CRP, values of eGFR below $80 \mathrm{~mL} / \mathrm{min}$ were associated with high ABI (RR 1.6; 95\% CI 1.0-2.3; $P=.03)$. Notably, these results were similar when CKD was defined by creatinine. Another study by Makita et al. [6] further expanded the observation by documenting that arterial stiffness increases in early CKD stages even in the absence of disease of the intimal layer of the arterial wall and independently of traditional CV risk factors [6].
The link between CKD and arterial stiffness is further complicated by the fact that arterial stiffness may promote CKD progression [7-9]. Though evidence is far from being conclusive, Tomiyama et al. [9] noted that higher brachialankle pulse wave velocity (baPWV) was associated with greater annual rate of eGFR decline in a large cohort of 2053 Japanese subjects. Every increase of $1 \mathrm{~m} / \mathrm{s}$ in baseline baPWV was associated with a $36 \%$ greater risk of having the GFR dropping below $60 \mathrm{~mL} / \mathrm{min}$ at followup (odds ratio 1.36; 95\% confidence interval: $1.09-1.70 ; P<.01)$. In contrast, baseline eGFR did not seem to be associated with the annual rate of change in baPWV suggesting that elevated arterial stiffness may also be an independent predictor of renal function decline [9].

\section{Arterial Stiffness as a Marker of CV Disease and Poor CV Prognosis}

As a marker of cardiovascular disease, increased arterial stiffness is linked to several other markers of CV dysfunction such as microalbuminuria and proteinuria, vascular calcification (VC), and left ventricular hypertrophy (LVH).

A few lines of evidence suggest that albuminuria, a recognized marker of vascular dysfunction, is associated with arterial stiffness. In a large observational study of 2180 patients, Liu and coworkers [10] noted a significant trend $(P<.001)$ toward a higher probability of having albuminuria associated with higher brachial-ankle pulse wave velocity (baPWV). When compared to the lowest quartile of baPWV, the adjusted odds ratios of having albuminuria during the followup were 1.12 (95\% CI: 0.63-2.02), 2.04 (95\% CI: 1.153.60 ), and 2.45 (95\% CI: 1.29-4.65) for the second, third, and fourth quartile of baPWV, respectively. Notably, the strength of the association between baPWV and microalbuminuria was not attenuated by multiple adjustments for potential confounders and was enhanced in diabetic and hypertensive patients [10].

A significant link between aortic PWV and vascular calcification burden has also been described in CKD patients. In a previous work [11], we showed that CKD patients receiving dialysis with great calcification burden in the abdominal aorta were more likely to have a significantly higher PWV. Adjustment for several confounders did not significantly affect this association [11]. In another paper by $\mathrm{Di}$ Iorio et al. [12], every 20 units increase in CACS corresponded to a significant $23 \%$ (95\% CI: $1.12-1.27$; $P<.001)$ increase in the risk of experiencing $1 \mathrm{~m} / \mathrm{sec}$ increase in PWV during follow-up. Interestingly the link between arterial stiffness and the chronic kidney diseasemineral bone disorder (CKD-MBD) syndrome might not be limited to vascular calcification. Indeed, data from the MultiEthnic Study of Atherosclerosis (MESA) study show that higher phosphorous levels are strongly associated with the ankle-brachial index (ABI). A phosphorous level $>4 \mathrm{mg} / \mathrm{dL}$ compared with $<3.0 \mathrm{mg} / \mathrm{dL}$ was associated with a significant fourfold increased risk for high ABI (>1.3)(relative risk : 4.6; 95\% CI 1.6-13.2; $P=.01$ ) [13]. 
A few cross-sectional studies [14-16] and a recent randomized controlled trial (RCT) [17] support the hypothesis of a strong connection between arterial stiffness and left ventricle and atrium remodeling. Indeed, lower arterial compliance increases aortic pulse pressure and the cardiac workload possibly leading to left ventricular hypertrophy (LVH) and dysfunction. Nitta and coworkers [14] documented a significant correlation between LVH and PWV $(r=0.44$, $P=.0014)$ in a small series of 49 patients receiving dialysis. A subsequent paper [18] showed in a cohort of CKD stage 2 and 3 patients that aortic distensibility and LV mass assessed by cardiac magnetic resonance were inversely associated $(r=$ $0.349, P<.01)$ [18]. Notably, examinations of patients and controls showed that arterial elastance (a measure of aortic stiffness) and end-systolic elastance (a measure of left ventricular stiffness) were significantly correlated $(r=0.692$, $P<.001)$ underscoring the association between arterial stiffness and cardiac function [18]. Finally, a recent RCT [17] documented that the addition of spironolactone on top of ACE inhibitors or angiotensin receptor blockers (ARBs) attenuates both left ventricular hypertrophy and arterial stiffness.

Low arterial compliance has been associated with an excess of morbidity and mortality in the general population and in CKD patients $[19,20]$. In a seminal study, Blacher and coworkers [20] documented that aortic stiffness determined by measurement of aortic PWV was linearly associated with all-cause and CV mortality in a cohort of 241 patients undergoing maintenance dialysis (adjusted odds ratio per $1 \mathrm{~m} / \mathrm{sec}$ increase in all-cause mortality: 1.39: 95\% CI, 1.19 to 1.62). Similar results were reached in a larger series of 1084 patients receiving dialysis recruited in the CORD study [21]. Indeed, each $1 \mathrm{~m} / \mathrm{s}$ increase in PWV was associated with a significant $15 \%$ higher risk of dying during the 24 months of follow-up [21]. Of interest is that adjusting for age, diabetes, and serum albumin did not attenuate these results [21].

\section{Therapeutic Approaches to Arterial Stiffness and Impact of Arterial Stiffness Attenuation on Survival}

A few therapeutic approaches have been attempted to reduce arterial stiffness both in the general population and in CKD patients.

Agents that modulate mineral metabolism abnormalities may positively affect arterial stiffness. Two small observational studies showed that the use of Sevelamer, a noncalcium-containing phosphate binder, may significantly attenuate PWV deterioration in CKD patients receiving chronic dialysis $[22,23]$. In another small series of 132 patients new to dialysis, the use of Sevelamer was associated with a significant reduction in the risk of PWV increase during the first 12 months of dialysis (odds ratio: 0.86; 95\%CI $0.83-0.91, P=.05)$ [12]. Cinacalcet may also reduce arterial stiffness in hemodialysis patients [24]. Indeed, Bonet and coworkers [24] in a small-scale prospective and observational study noted that cinacalcet administration to 21 patients resulted in a significant reduction of PWV after
12 months $(9.35(1.83)$ versus $8.66(1.86) \mathrm{m} / \mathrm{sec} ; P=.03$; before and after treatment).

Some preliminary data suggest that Lipid lowering agents may also lower arterial stiffness. In a recent RCT [25], 37 CKD stage 2-4 patients were selected to receive $10 \mathrm{mg}$ atorvastatin or placebo for 3 years. At study completion, aortic PWV showed a significant increase over time in placebo-treated but not in atorvastatin-treated patients $(0.51$ $(0.95)$ versus $0.30(0.75) \mathrm{m} / \mathrm{sec}$ per year in placebo- and atorvastatin-treated patients, resp.) [25]. Of importance is that these promising results were not confirmed in an ancillary study entitled the Anglo-Scandinavian Cardiac Outcomes Trial (ASCOT) [26]. In this study, 891 hypertensive patients were randomized to atorvastatin $10 \mathrm{mg} /$ day or placebo and followedup for a mean time of 3.5 years. Contrary to what was documented among CKD patients, authors could not detect any effect of statin on arterial stiffness [26].

The effect of antihypertensive agents acting on the reninangiotensin-aldosterone system (RAAS) has also been tested. Edwards and coworkers [17] randomized 112 patients with stage 2 and $3 \mathrm{CKD}$ and a good blood pressure control with ACE inhibitors or angiotensin receptor blockers (ARBs) to spironolactone $(25 \mathrm{mg})$ or placebo. After 40 weeks of treatment, the use of spironolactone reduced both arterial stiffness and left ventricular mass [17].

The role of RAAS on arterial system was also suggested in a previous study of 2199 hypertensive patients. When compared to the association of atenolol and thiazide, amlodipine and perindopril showed a superior reduction of aortic pulse pressure (an index of arterial stiffness) despite a similar impact on brachial blood pressure [27]. Providing that aortic pulse pressure was significantly associated with the composite outcome of cardiovascular events and the development of renal impairment [27], these results suggest that central artery stiffness attenuation may significantly improve survival. In this regard, a study by Guerin and coworker [28] further corroborates this hypothesis. In this study, the absence of PWV decrease with treatment with ACE-I was an independent predictor of all-cause and CV mortality during follow-up [28].

\section{Conclusion}

Convincing evidence supports the notion that arterial stiffness is a marker of arterial damage and associated with an adverse prognosis. These concepts have been validated in the general population as well as in CKD patients. Although screening for arterial stiffness increase in patients with CKD might not fulfill the WHO's suggested requirements for early disease detection [29], preliminary evidence indicates that the integration of demographic and clinical characteristics with information derived from arterial stiffness assessment may represent an accurate and cost-effective approach for individualizing patients' care and treatment. Finally, preliminary evidence shows that attenuation of arterial stiffness may be associated with a significant improvement in survival. 


\section{References}

[1] T. J. Wang, P. Gona, M. G. Larson et al., "Multiple biomarkers for the prediction of first major cardiovascular events and death," New England Journal of Medicine, vol. 355, no. 25, pp. 2631-2639, 2006.

[2] A. S. Go, G. M. Chertow, D. Fan, C. E. McCulloch, and C. Y. Hsu, "Chronic kidney disease and the risks of death, cardiovascular events, and hospitalization," New England Journal of Medicine, vol. 351, no. 13, pp. 1296-1305, 2004.

[3] M. G. Shlipak, R. Katz, M. J. Sarnak et al., "Cystatin C and prognosis for cardiovascular and kidney outcomes in elderly persons without chronic kidney disease," Annals of Internal Medicine, vol. 145, no. 4, pp. 237-246, 2006.

[4] M. C. Wang, W. C. Tsai, JU. Y. Chen, and J. J. Huang, "Stepwise increase in arterial stiffness corresponding with the stages of chronic kidney disease," American Journal of Kidney Diseases, vol. 45, no. 3, pp. 494-501, 2005.

[5] J. H. Ix, R. Katz, I. H. De Boer et al., "Association of chronic kidney disease with the spectrum of ankle brachial index the CHS (Cardiovascular Health Study)," Journal of the American College of Cardiology, vol. 54, no. 13, pp. 1176-1184, 2009.

[6] S. Makita, A. Abiko, Y. Naganuma, M. Nagai, and M. Nakamura, "Chronic kidney disease is associated with increased carotid artery stiffness without morphological changes in participants of health check-up programs," Atherosclerosis, vol. 213, no. 1, pp. 306-310, 2010.

[7] M. L. Ford, L. A. Tomlinson, T. P. E. Chapman, C. Rajkumar, and S. G. Holt, "Aortic stiffness is independently associated with rate of renal function decline in chronic kidney disease stages 3 and 4," Hypertension, vol. 55, no. 5, pp. 1110-1115, 2010.

[8] C. D. Chue, N. C. Edwards, L. J. Davis, R. P. Steeds, J. N. Townend, and C. J. Ferro, "Serum phosphate but not pulse wave velocity predicts decline in renal function in patients with early chronic kidney disease," Nephrology Dialysis Transplantation. In press.

[9] H. Tomiyama, H. Tanaka, H. Hashimoto et al., "Arterial stiffness and declines in individuals with normal renal function/early chronic kidney disease," Atherosclerosis, vol. 212, no. 1, pp. 345-350, 2010.

[10] C.-S. Liu, F. X. Pi-Sunyer, C.-I. Li et al., "Albuminuria is strongly associated with arterial stiffness, especially in diabetic or hypertensive subjects-a population-based study (Taichung Community Health Study, TCHS)," Atherosclerosis, vol. 211, no. 1, pp. 315-321, 2010.

[11] P. Raggi, A. Bellasi, E. Ferramosca, T. Islam, P. Muntner, and G. A. Block, "Association of pulse wave velocity with vascular and valvular calcification in hemodialysis patients," Kidney International, vol. 71, no. 8, pp. 802-807, 2007.

[12] B. Di Iorio, O. Nargi, E. Cucciniello et al., "Coronary artery calcification progression is associated with arterial stiffness and cardiac repolarization deterioration in hemodialysis patients," Kidney and Blood Pressure Research, vol. 34, no. 3, pp. 180-187, 2011.

[13] J. H. Ix, I. H. De Boer, C. A. Peralta et al., "Serum phosphorus concentrations and arterial stiffness among individuals with normal kidney function to moderate kidney disease in MESA," Clinical Journal of the American Society of Nephrology, vol. 4, no. 3, pp. 609-615, 2009.

[14] K. Nitta, T. Akiba, K. Uchida et al., "Left ventricular hypertrophy is associated with arterial stiffness and vascular calcification in hemodialysis patients," Hypertension Research, vol. 27, no. 1, pp. 47-52, 2004.
[15] M. C. Wang, W. C. Tsai, J. Y. Chen, M. F. Cheng, and J. J. Huang, "Arterial stiffness correlated with cardiac remodelling in patients with chronic kidney disease," Nephrology, vol. 12, no. 6, pp. 591-597, 2007.

[16] G. M. London, S. J. Marchais, A. P. Guerin, F. Metivier, H. Adda, and B. Pannier, "Inflammation, arteriosclerosis, and cardiovascular therapy in hemodialysis patients," Kidney International, Supplement, vol. 63, no. 84, pp. S88-S93, 2003.

[17] N. C. Edwards, R. P. Steeds, P. M. Stewart, C. J. Ferro, and J. N. Townend, "Effect of spironolactone on left ventricular mass and aortic stiffness in early-stage chronic kidney disease: a randomized controlled trial," Journal of the American College of Cardiology, vol. 54, no. 6, pp. 505-512, 2009.

[18] N. C. Edwards, C. J. Ferro, J. N. Townend, and R. P. Steeds, "Aortic distensibility and arterial-ventricular coupling in early chronic kidney disease: a pattern resembling heart failure with preserved ejection fraction," Heart, vol. 94, no. 8, pp. 10381043, 2008.

[19] T. W. Hansen, J. A. Staessen, C. Torp-Pedersen et al., "Prognostic value of aortic pulse wave velocity as index of arterial stiffness in the general population," Circulation, vol. 113, no. 5, pp. 664-670, 2006.

[20] J. Blacher, A. P. Guerin, B. Pannier, S. J. Marchais, M. E. Safar, and G. M. London, "Impact of aortic stiffness on survival in end-stage renal disease," Circulation, vol. 99, no. 18, pp. 24342439, 1999.

[21] F. Verbeke, W. Van Biesen, E. Honkanen et al., "Prognostic value of aortic stiffness and calcification for cardiovascular events and mortality in dialysis patients: outcome of the Calcification Outcome in Renal Disease (CORD) study," Clinical Journal of the American Society of Nephrology, vol. 6, no. 1, pp. 153-159, 2011.

[22] T. E. H. Othmane, G. Bakonyi, J. Egresits et al., "Effect of sevelamer on aortic pulse wave velocity in patients on hemodialysis: a prospective observational study," Hemodialysis International, vol. 11, no. 3, pp. S13-S21, 2007.

[23] T. Takenaka and H. Suzuki, "New strategy to attenuate pulse wave velocity in haemodialysis patients," Nephrology Dialysis Transplantation, vol. 20, no. 4, pp. 811-816, 2005.

[24] J. Bonet, B. Bayés, P. Fernández-Crespo, M. Casals, J. LópezAyerbe, and R. Romero, "Cinacalcet may reduce arterial stiffness in patients with chronic renal disease and secondary hyperparathyroidism - results of a small-scale, prospective, observational study," Clinical Nephrology, vol. 75, no. 3, pp. 181-187, 2011.

[25] R. G. Fassett, I. K. Robertson, M. J. Ball, D. P. Geraghty, J. E. Sharman, and J. S. Coombes, "Effects of atorvastatin on arterial stiffness in chronic kidney disease: a randomised controlled trial," Journal of Atherosclerosis and Thrombosis, vol. 17, no. 3, pp. 235-241, 2010.

[26] B. Williams, P. S. Lacy, J. K. Cruickshank et al., "Impact of statin therapy on central aortic pressures and hemodynamics: principal results of the Conduit Artery Function EvaluationLipid-Lowering Arm (CAFE-LLA) study," Circulation, vol. 119, no. 1, pp. 53-61, 2009.

[27] B. Williams, P. S. Lacy, S. M. Thom et al., "Differential impact of blood pressure-lowering drugs on central aortic pressure and clinical outcomes: principal results of the Conduit Artery Function Evaluation (CAFE) study," Circulation, vol. 113, no. 9, pp. 1213-1225, 2006.

[28] A. P. Guerin, J. Blacher, B. Pannier, S. J. Marchais, M. E. Safar, and G. M. London, "Impact of aortic stiffness attenuation on survival of patients in end-stage renal failure," Circulation, vol. 103, no. 7, pp. 987-992, 2001. 
[29] K. Strong, N. Wald, A. Miller, and A. Alwan, "Current concepts in screening for noncommunicable disease: World Health Organization Consultation Group Report on methodology of noncommunicable disease screening," Journal of Medical Screening, vol. 12, no. 1, pp. 12-19, 2005. 


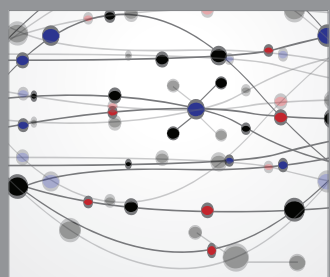

The Scientific World Journal
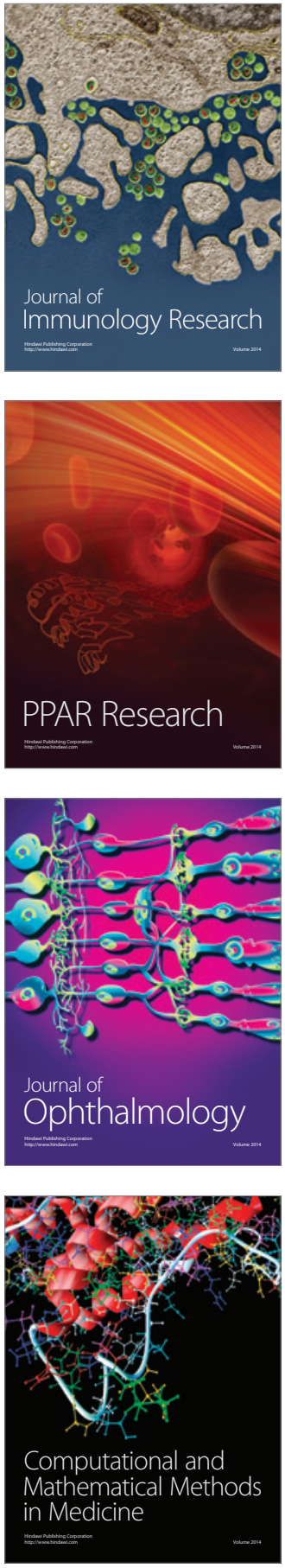

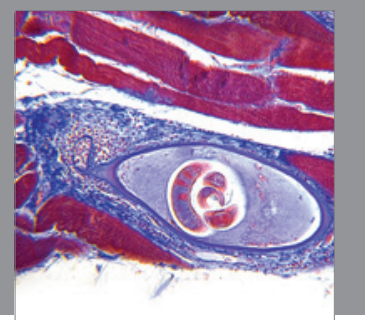

Gastroenterology

Research and Practice
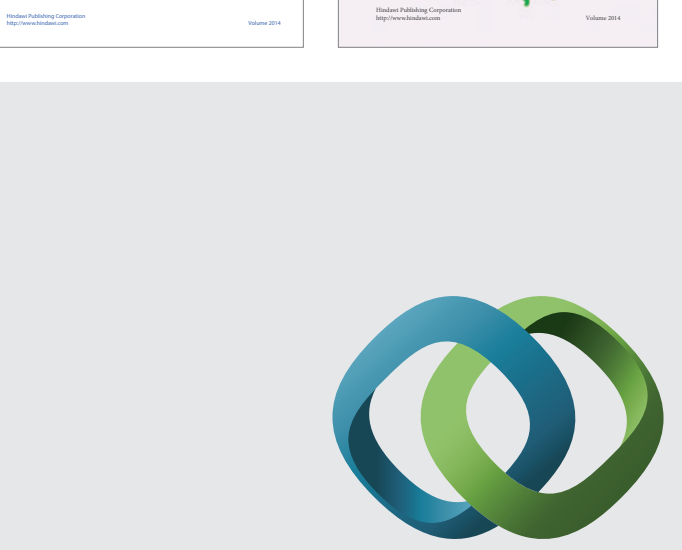

\section{Hindawi}

Submit your manuscripts at

http://www.hindawi.com
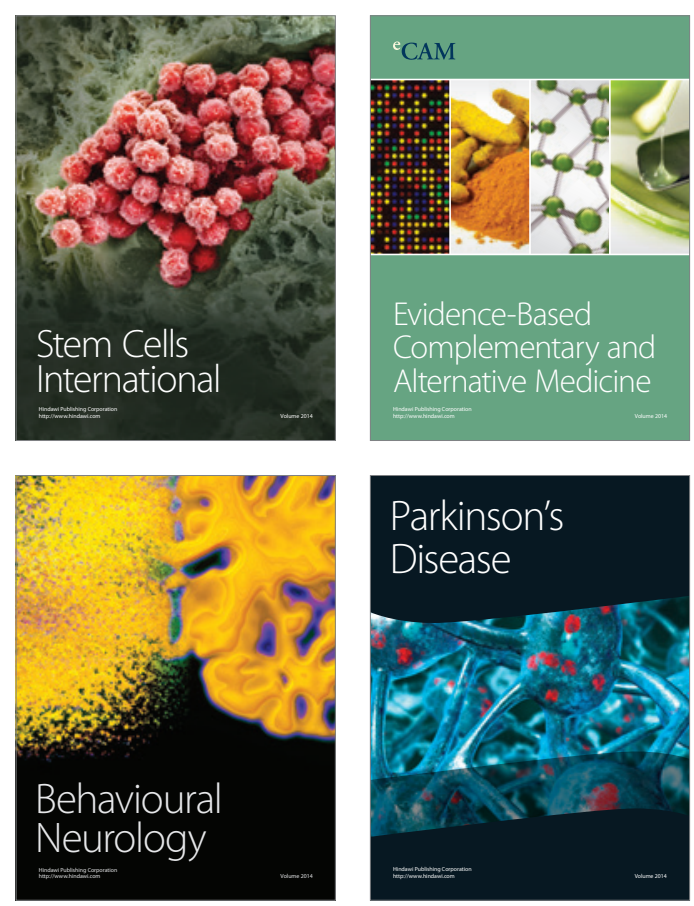

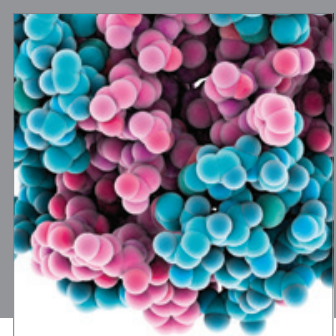

Journal of
Diabetes Research

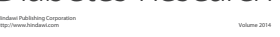

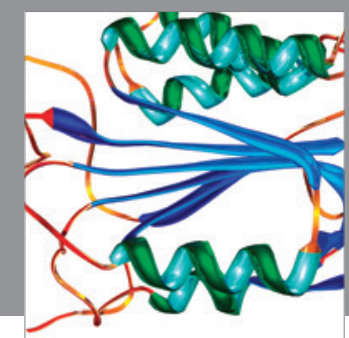

Disease Markers
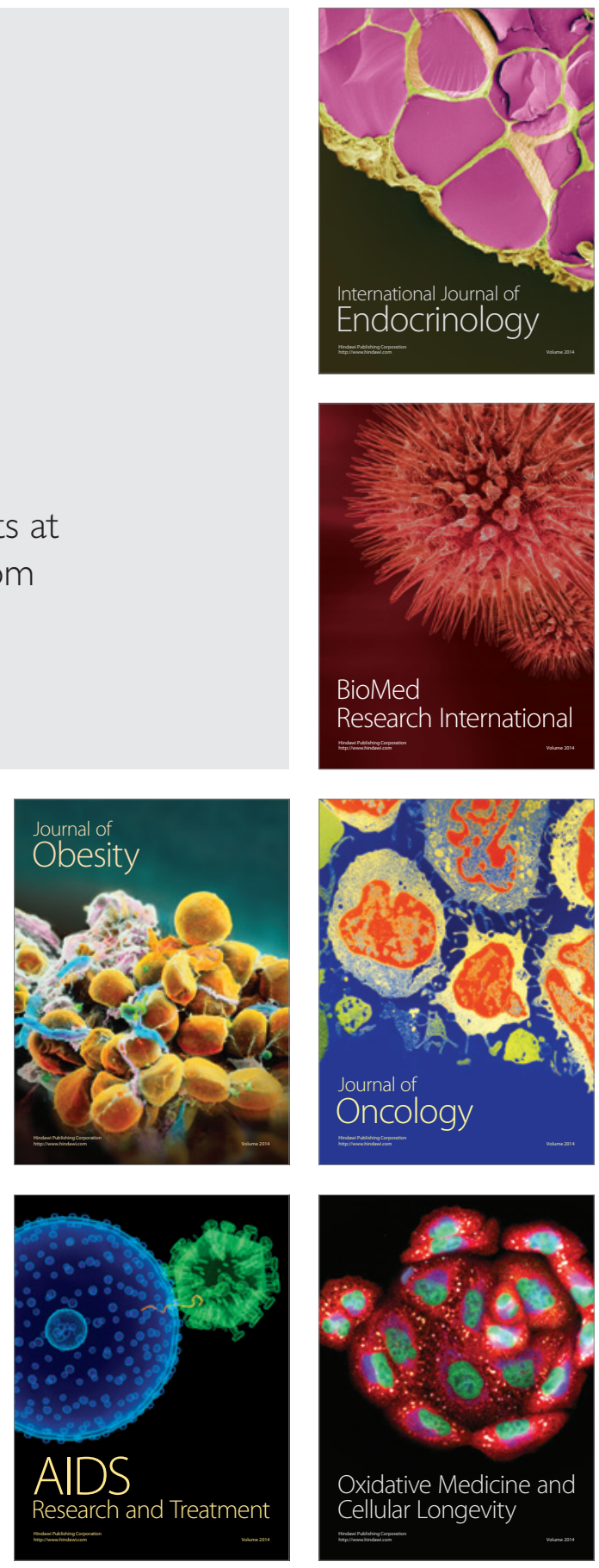